\title{
FINITARY AXIOMATIZATIONS OF THE TRUE RELATIONAL EQUATIONS
}

\author{
ROGER D. MADDUX \\ Department of Mathematics, Iowa State University \\ Ames, Iowa 50011, U.S.A.
}

1. Introduction. Y. Venema $[20,21]$ has shown that if an equation is true in every representable relation algebra, then it can be derived, by means of the usual deductive rules of equational logic together with a special rule, from any finite set of axioms for the class of relation algebras. The special rule can take any of the following forms, in which $x$ is a relation-algebraic term, and $v$ is a variable which does not occur in $x$ :

$$
\begin{array}{cccc}
\text { (C) } & \frac{(0 \dagger v) \cdot\left(0 \dagger v \dagger 1^{\prime}\right) \cdot x=0}{x=0} & \frac{1 ; \bar{v}+1 ; v ; 0^{\prime}+x=1}{x=1} \\
\left(\mathrm{C}^{\prime}\right) & \frac{v \cdot\left(1^{\prime} \dagger \bar{v} \dagger 0^{\prime}\right) \cdot\left(0 \dagger \bar{v} \dagger 1^{\prime}\right) \cdot x=0}{x=0} & \frac{\bar{v}+0^{\prime} ; v ; 1+1 ; v ; 0^{\prime}+x=1}{x=1}
\end{array}
$$

Rule (C) is used in [20] and is given here first in its Boolean dual form. A slight variant of the second version of rule $\left(\mathrm{C}^{\prime}\right)$ is used in [21]. These results will be improved here in two ways. First, with any of the rules above it suffices to use an axiomatization of the semiassociative relation algebras instead of the relation algebras: this amounts to replacing the associative law by the semiassociative law. The other improvement is that the rules above can be replaced by the following rule (accompanied by its Boolean dual), where, again, $x$ is a term and $v$ is a variable which does not occur in $x$ :

$$
\frac{v \cdot\left(\bar{v} \dagger 1^{\prime}\right) \cdot x=0}{x=0} \quad \frac{\bar{v}+v ; 0^{\prime}+x=1}{x=1}
$$

It is not possible to do both improvements: one of the two simple rules above together with the axioms for semiassociative relation algebras are not enough to derive all the equations true in all representable relation algebras.

1991 Mathematics Subject Classification: 03G15.

The paper is in final form and no version of it will be published elsewhere. 
It follows from any of these results that the equational theory of representable relation algebra is recursively enumerable, and hence, by an equational variant of "Craig's Trick", has a recursive equational axiomatization. Lyndon [7] gave an explicit recursive equational axiomatization, and McKenzie [16] (see also [18]) found a simpler proof that such an axiomatization exists.

For general information and introductory material on relation algebras and semiassociative relation algebras the following references are recommended: [1], $[2],[3, \S 5.3],[4],[5],[6],[10],[12],[13],[14]$, and [19].

2. Equational logic. Let $\mathbf{V}=\left\{\mathbf{v}_{n}: n \in \omega\right\}$. The elements of $\mathbf{V}$ are called variables. Assume that $\mathbf{v}_{n} \neq \mathbf{v}_{m}$ whenever $n \neq m$. Let

$$
\mathfrak{T}=\left\langle T,+, \cdot,-, 0,1, \dagger, ;,^{\smile}, 0^{\prime}, 1^{\prime}\right\rangle
$$

be an algebra with universe $T$, four binary operations $+, \cdot, \dagger$, and ; two unary operations ${ }^{-}$and ' , and four constants 1, 0, 1', and 0', which is absolutely freely generated by $\mathbf{V}$. The elements of $T$ are called terms, and $\mathfrak{T}$ is called the algebra of terms. An equation is simply an ordered pair of terms. Equations will be written $x=y$, where $x$ and $y$ are terms, instead of $\langle x, y\rangle$. Thus, for all $x, y, u, v \in T$, $x=y=u=v$ iff $x=u$ and $y=v$. Parentheses are omitted from expressions denoting terms according to the convention that unary operations are performed first, followed by $;, \cdot, \dagger$, and + , in that order. The computation of repeated binary operations is from left to right.

Let $\mathfrak{A}$ be any algebra with the same similarity type as $\mathfrak{T}$. The assumption that $\mathfrak{T}$ is absolutely freely generated by $\mathbf{V}$ means that if $h$ is any function which maps $\mathbf{V}$ into $\mathfrak{A}$, then there is a unique extension of $h$ to a homomorphism from $\mathfrak{T}$ into $\mathfrak{A}$. An equation $x=y$ is true in $\mathfrak{A}$ (in symbols, $\mathfrak{A} \vDash x=y$ ) if $h(x)=h(y)$ for every homomorphism $h$ from $\mathfrak{T}$ into $\mathfrak{A}$. A set $\Sigma$ of equations (that is, a binary relation on $T$ ) is true in $\mathfrak{A}$ (in symbols, $\mathfrak{A} \vDash \Sigma$ ) if $\mathfrak{A} \vDash x=y$ for every $x=y \in \Sigma$. If $K$ is any class of such algebras similar to $\mathfrak{T}$, then the equation $x=y$ is true in $K$ (symbolically, $K \vDash x=y$ ) if $\mathfrak{A} \vDash x=y$ for every $\mathfrak{A} \in K$.

A set $\Omega$ of equations is deductively closed if the following conditions hold:

$\left(\mathrm{R}_{1}\right) \quad$ If $h$ is a homomorphism from $\mathfrak{T}$ into $\mathfrak{T}$, and $x=y \in \Omega$, then $h(x)=h(y)$ $\in \Omega$.

$\left(\mathrm{R}_{2}\right) \quad x=x \in \Omega$ for every $x \in T$.

$\left(\mathrm{R}_{3}\right) \quad$ If $x=y, x=z \in \Omega$ then $y=z \in \Omega$.

$\left(\mathrm{R}_{4}\right) \quad$ If $x=y \in \Omega$ and $z \in T$, then the following equations are also in $\Omega$ :

$$
\begin{aligned}
x+z & =y+z, & z+x & =z+y, \\
x \cdot z & =y \cdot z, & z \cdot x & =z \cdot y, \\
\bar{x} & =\bar{y}, & \breve{x} & =\breve{y}, \\
x \dagger z & =y \dagger z, & z \dagger x & =z \dagger y, \\
x ; z & =y ; z, & z ; x & =z ; y .
\end{aligned}
$$


For any set $\Psi$ of equations, and any equation $\psi$, we say that $\psi$ is equationally deducible from $\Psi$, in symbols $\Psi \vdash \psi$, if $\psi$ belongs to every deductively closed set of equations which includes $\Psi$.

3. Semiassociative relation algebras and relation algebras. We define the classes of relation algebras and semiassociative relation algebras by giving axiom sets for them. Let $\Xi_{\text {SA }}$ be the set which contains the following equations.

$$
\begin{aligned}
& \mathbf{v}_{0}+\mathbf{v}_{1}=\mathbf{v}_{1}+\mathbf{v}_{0}, \\
& \mathbf{v}_{0}+\left(\mathbf{v}_{1}+\mathbf{v}_{2}\right)=\left(\mathbf{v}_{0}+\mathbf{v}_{1}\right)+\mathbf{v}_{2}, \\
& \overline{\overline{\mathbf{v}_{0}}+\mathbf{v}_{1}}+\overline{\overline{\mathbf{v}_{0}}+\overline{\mathbf{v}_{1}}}=\mathbf{v}_{0} \text {, } \\
& \left(\mathbf{v}_{0} ; 1\right) ; 1=\mathbf{v}_{0} ; 1 \text {, } \\
& \left(\mathbf{v}_{0}+\mathbf{v}_{1}\right) ; \mathbf{v}_{2}=\mathbf{v}_{0} ; \mathbf{v}_{2}+\mathbf{v}_{1} ; \mathbf{v}_{2} \text {, } \\
& \mathbf{v}_{0} ; 1^{\prime}=\mathbf{v}_{0} \text {, } \\
& \left(\breve{\mathbf{v}_{0}}\right)^{\smile}=\mathbf{v}_{0}, \\
& \left(\mathbf{v}_{0}+\mathbf{v}_{1}\right)^{\smile}=\breve{\mathbf{v}_{0}}+\breve{\mathbf{v}_{1}} \text {, } \\
& \left(\mathbf{v}_{0} ; \mathbf{v}_{1}\right)^{\smile}=\breve{\mathbf{v}_{1}} ; \breve{\mathbf{v}_{0}} \text {, } \\
& \breve{\mathbf{v}_{0}} ; \overline{\mathbf{v}_{0} ; \mathbf{v}_{1}}+\overline{\mathbf{v}_{1}}=\overline{\mathbf{v}_{1}} \text {, } \\
& \mathbf{v}_{0} \cdot \mathbf{v}_{1}=\overline{\overline{\mathbf{v}_{0}}+\overline{\mathbf{v}_{1}}} \text {, } \\
& \mathbf{v}_{0} \dagger \mathbf{v}_{1}=\overline{\overline{\mathbf{v}_{0}} ; \overline{\mathbf{v}_{1}}} \text {, } \\
& 0^{\prime}=\overline{1^{\prime}} \text {, } \\
& 1=1^{\prime}+0^{\prime}, \\
& 0=\overline{1} \text {. }
\end{aligned}
$$

$\Xi_{\mathrm{SA}}$ is called the set of axioms for semiassociative relation algebras. Also, let $\Xi_{\mathrm{RA}}=\Xi_{\mathrm{SA}} \cup\left\{\mathbf{v}_{0} ;\left(\mathbf{v}_{1} ; \mathbf{v}_{2}\right)=\left(\mathbf{v}_{0} ; \mathbf{v}_{1}\right) ; \mathbf{v}_{2}\right\} . \Xi_{\mathrm{RA}}$ is called the set of axioms for relation algebras. Let $\mathfrak{A}$ be an algebra similar to $\mathfrak{T} . \mathfrak{A}$ is a semiassociative relation algebra if $\mathfrak{A} \vDash \Xi_{\mathrm{SA}}$, and $\mathfrak{A}$ is a relation algebra if $\mathfrak{A} \vDash \Xi_{\mathrm{RA}}$. SA and RA are the classes of semiassociative relation algebras and relation algebras, respectively. On the basis of these definitions, we get the following results by the standard methods of equational logic.

THEOREM 1. For all terms $x, y \in T, \mathrm{SA} \vDash x=y$ iff $\Xi_{\mathrm{SA}} \vdash x=y$.

TheOREM 2. For all terms $x, y \in T, \mathrm{RA} \vDash x=y$ iff $\Xi_{\mathrm{RA}} \vdash x=y$.

4. Proper and representable relation algebras. Let $\mathfrak{A}$ be an algebra similar to $\mathfrak{T}$. Then $\mathfrak{A}$ is a proper relation algebra if the universe $A$ of $\mathfrak{A}$ consists entirely of binary relations, the binary relation 1 ' is the identity relation on the field of 1 (the largest binary relation in $A$ ), $\mathfrak{A}$ is a semiassociative relation algebra (which ensures that certain identities hold), and, for all relations $x, y \in A, x+y$ is the union of $x$ and $y, x \cdot y$ is the intersection of $x$ and $y, \bar{x}$ is the complement of $x$ 
with respect to $1, x \dagger y$ is the relative sum of $x$ and $y, x ; y$ is the relative product of $x$ and $y$, and $\breve{x}$ is the converse of $x$. $\mathfrak{A}$ is a representable relation algebra if $\mathfrak{A}$ is isomorphic to a proper relation algebra. RRA is the class of all representable relation algebras.

Since $\Xi_{\mathrm{SA}}$ and $\Xi_{\mathrm{RA}}$ are finite sets, we say that SA and RA are finitely based. However, RRA is not finitely based [17]. This can be expressed as follows.

TheOrem 3 [17]. Let $\Sigma$ be a set of equations. Suppose, for all terms $x, y \in T$, that RRA $\vDash x=y$ iff $\Sigma \vdash x=y$. Then $\Sigma$ is infinite.

5. Special elements. Let $\mathfrak{A}$ be an algebra similar to $\mathfrak{T}$. An element $x \in A$ is functional if $x \cdot x ; 0^{\prime}=0$, and constant-functional if $1 ; x \cdot x ; 0^{\prime}=0$. For every $v \in A$ let $f(v)=v \cdot\left(\bar{v} \dagger 1^{\prime}\right)$, and let $c(v)=(0 \dagger v) \cdot\left(0 \dagger \bar{v} \dagger 1^{\prime}\right)$. If $\mathfrak{A}$ is a semiassociative relation algebra, then $c(v)=0 \dagger\left(v \cdot\left(\bar{v} \dagger 1^{\prime}\right)\right)=0 \dagger f(v)$, and, by the cycle law [12, Def. 1], $x$ is functional iff $\breve{x} ; x \leq 1$ ', so our definition coincides with the original definition of a functional element in a relation algebra [1, Def. 3.34], and $x$ is constant-functional iff $\breve{x} ; 1 ; x \leq 1$ '. In a proper relation algebra, functional elements are functions, and constant-functional elements are constant functions.

LEMMA. If $v$ is an element of a semiassociative relation algebra, then $f(v)$ is functional and $c(v)$ is constant-functional.

Pro of. The functionality of $f(v)$ actually holds in every nonassociative relation algebra (defined in [10]). Indeed, we have $f(v) \leq v$ and $f(v) ; 0^{\prime} \leq\left(\bar{v} \dagger 1^{\prime}\right) ; 0^{\prime} \leq$ $\bar{v}$ by $[10$, Th. $1.4(1)]$, so $f(v) \cdot f(v) ; 0^{\prime}=0$. To prove that $c(v)$ is constant-functional we need one of the special laws holding in semiassociative relation algebras:

$$
\begin{array}{rlrl}
1 ; c(v) \cdot c(v) ; 0^{\prime} & =1 ;(0 \dagger f(v)) \cdot(0 \dagger f(v)) ; 0 & & \\
& \leq 1 ;(0 \dagger v) \cdot\left(0 \dagger\left(\bar{v} \dagger 1^{\prime}\right)\right) ; 0 & & \\
& =1 ;(0 \dagger v) \cdot\left(0 \dagger \bar{v} \dagger 1^{\prime}\right) ; 0 & & {[12, \text { Th. } 13(\mathrm{iv})]} \\
& \leq v \cdot(0 \dagger \bar{v}) & & {[10, \text { Th. } 1.4(1)(2)]} \\
& \leq v \cdot \bar{v} & & \\
& =0 .
\end{array}
$$

We will use two representation results involving these special kinds of elements.

Theorem 4 ([15], [8, Cor. 9]). If $\mathfrak{A} \in \mathrm{RA}$ and every nonzero element of $\mathfrak{A}$ contains a functional element, then $\mathfrak{A}$ is a representable relation algebra.

THEOREM 5. If $\mathfrak{A} \in \mathrm{SA}$ and every nonzero element of $\mathfrak{A}$ contains a nonzero constant-functional element, then $\mathfrak{A}$ is a representable relation algebra.

P r o of. Assume $\mathfrak{A} \in \mathrm{SA}$ and every nonzero element of $\mathfrak{A}$ contains a nonzero constant-functional element. We will show that $\mathfrak{A}$ is point-dense [12, Def. 44], that is, every nonzero element below 1' contains a point. Suppose $0 \neq x \leq 1$ '. By hypothesis, there is some $y \in A$ such that $0 \neq y \leq x$ and $1 ; y \cdot y ; 0$ ' $=0$. From 
$y \leq x$ and $x \leq 1$ ' we get $y \leq 1$ '. Then

$$
\begin{aligned}
0 & =1 ; y \cdot y ; 0 & & \\
& =y ;(1 ; y) \cdot 0, & & {[11, \text { Lem. } 5(\mathrm{i})] } \\
& =y ; 1 ; y \cdot 0, & & {[11, \text { Lem. } 5(\mathrm{iv})] }
\end{aligned}
$$

so $y ; 1 ; y \leq 1$. Thus $y$ is a point [12, Def. $38(i i)]$. Since $\mathfrak{A}$ is a point-dense semiassociative relation algebra, it must be a representable relation algebra $[12$, Th. 54].

6. Special deductive rules. We say that a variable $\mathbf{v}_{n}$ does not occur in a term $x \in T$ if $x$ belongs to the subalgebra of $\mathfrak{T}$ which is generated by $\left\{\mathbf{v}_{k}: k \neq n\right\}$. A set $\Omega$ of equations is c-closed if $\Omega$ is deductively closed and

(C) if $x \in T, v \in \mathbf{V}, v$ does not occur in $x$, and $c(v) \cdot x=0 \in \Omega$, then $x=0 \in \Omega$. Similarly, $\Omega$ is $f$-closed if $\Omega$ is deductively closed and

(F) $\quad$ if $x \in T, v \in \mathbf{V}, v$ does not occur in $x$, and $f(v) \cdot x=0 \in \Omega$, then $x=0 \in \Omega$.

For any set $\Psi$ of equations, and any equation $\psi$, we say that $\psi$ is $c$-deducible from $\Psi$, in symbols $\Psi \vdash^{\mathrm{c}} \psi$, if $\psi$ belongs to every c-closed set of equations containing $\Psi$, and $\psi$ is $f$-deducible from $\Psi$, in symbols $\Psi \vdash^{\mathrm{f}} \psi$, if $\psi$ belongs to every f-closed set of equations containing $\Psi$.

With these notions it is now possible to give a precise statement of one of Venema's results indicated in the Introduction.

Theorem 6 [20, Th. 4.3]. $\Xi_{\mathrm{RA}}{ }^{\mathrm{c}} x=y$ iff RRA $\vDash x=y$.

In connection with Theorem 6 see also [21, Th. 3.5.11], which is essentially the same theorem, except that rule $\left(\mathrm{C}^{\prime}\right)$ is used instead of $(\mathrm{C})$. Now we proceed to our two improvements.

THEOREM 7. $\Xi_{\mathrm{RA}} \vdash^{\mathrm{f}} x=y$ iff RRA $\vDash x=y$.

Proof. For every term $x \in T$, let $[x]=\left\{y: \Xi_{\mathrm{RA}}{ }^{\mathrm{f}} x=y\right\}$, and, for any $x, y \in T$, define $x \cong y$ to mean $\Xi_{\mathrm{RA}} \vdash^{\mathrm{f}} x=y$. Then $\cong$ is a congruence relation on $\mathfrak{T}$ (by an easy proof involving only the rules $\left(\mathrm{R}_{2}\right)-\left(\mathrm{R}_{4}\right)$ in the definition of $\vdash^{\mathrm{f}}$ ), so there is a quotient algebra $\mathfrak{T} / \cong$. We have $\mathfrak{T} / \cong \in$ RA because we used $\Xi_{\text {RA }}$ in the definition of $\cong$.

Next we show that every nonzero element of $\mathfrak{T} / \cong$ contains a nonzero functional element. Assume $[x] \neq 0$ for some $x \in T$. By definition, this means that $\nvdash^{\mathrm{f}} x=0$. Choose a variable $v \in \mathbf{V}$ so that $v$ does not occur in $x$. Now if $\vdash^{\mathrm{f}} f(v) \cdot x=0$, then $\vdash^{\mathrm{f}} x=0$ by $(\mathrm{F})$, contradicting $\nvdash^{\mathrm{f}} x=0$. Thus we have $\nvdash^{\mathrm{f}} f(v) \cdot x=0$. It follows that $f([v]) \cdot[x]=[f(v) \cdot x] \neq 0$ in $\mathfrak{T} / \cong$. By the Lemma above, $f([v]) \cdot[x]$ is a functional element of $\mathfrak{T} / \cong$ which lies below $[x]$ in $\mathfrak{T} / \cong$.

Since every nonzero element of $\mathfrak{T} / \cong$ contains a nonzero functional element, we conclude, by Theorem 4 , that $\mathfrak{T} / \cong$ is a representable relation algebra. 
Now suppose that $x, y \in T$ and RRA $\vDash x=y$. Consider the map which sends every variable $\mathbf{v}_{n}$ to $\left[\mathbf{v}_{n}\right]$. This map has a unique extension to a homomorphism $h$ from $\mathfrak{T}$ onto $\mathfrak{T} / \cong$, such that $h(t)=[t]$ for every $t \in T$. In particular, we have $h(x)=[x]$ and $h(y)=[y]$, but $\mathfrak{T} / \cong \vDash x=y$ since $\mathfrak{T} / \cong \in \operatorname{RRA}$, so $[x]=[y]$, which implies that $\vdash^{\mathrm{f}} x=y$.

This completes the proof of half of the theorem, namely, if RRA $\vDash x=y$ then $\Xi_{\mathrm{RA}}{ }^{\mathrm{f}} x=y$.

Now we wish to show that if $\Xi_{\mathrm{RA}} \vdash^{\mathrm{f}} x=y$, then RRA $\vDash x=y$. Clearly it suffices to show that $(\mathrm{F})$ is sound, that is, if $\mathrm{RRA} \vDash f(v) \cdot x=0$ and $v$ does not occur in $x$, then $\operatorname{RRA} \vDash x=0$. Assume RRA $\vDash f(v) \cdot x=0$. Since RRA is the class of algebras isomorphic to proper relation algebras, we need only show $x=0$ holds in all proper relation algebras. Let $\mathfrak{A}$ be a proper relation algebra. To show $\mathfrak{A} \vDash x=0$, consider an arbitrary map of the variables of $x$ to relations in $\mathfrak{A}$, sending the term $x$ to the relation $X$. Every nonempty relation contains a nonempty function, so let $V$ be a function included in $X$ such that $V$ is nonempty only if $X$ is nonempty. Since $v$ does not occur in $x$, we may extend the map of the variables of $x$ to one which sends $v$ to $V$. This extended map still sends $x$ to $X$. Now $f(v) \cdot x=0$ is true in $\mathfrak{A}$, so this extended map sends $f(v) \cdot x$ to the empty relation. Thus $V \cap\left(\bar{V} \dagger 1^{\prime}\right) \cap X=\emptyset$, so $V \cap\left(\bar{V} \dagger 1^{\prime}\right) \subseteq \bar{X}$. Now $V$ is a function, so $V \subseteq \bar{V} \dagger 1^{\prime}$, hence $V \subseteq \bar{X}$. But $V \subseteq X$, so $V=\emptyset$. By the choice of $V$, this gives $X=\emptyset$, as desired.

\section{THEOREM 8. $\Xi_{\mathrm{SA}} \vdash^{\mathrm{c}} x=y$ iff $\mathrm{RRA} \vDash x=y$.}

The proof of this theorem is similar to the proof of Theorem 7 , so we only indicate the differences. For every term $x \in T$, let $[x]=\left\{y: \Xi_{\mathrm{SA}} \vdash^{\mathrm{f}} x=y\right\}$, and, for any $x, y \in T$, define $x \cong y$ to mean $\Xi_{\mathrm{SA}}{ }^{{ }^{\mathrm{f}}} x=y$. Then $\cong$ is a congruence relation on $\mathfrak{T}$ and $\mathfrak{T} / \cong \in \mathrm{SA}$.

Next we show that every nonzero element of $\mathfrak{T} / \cong$ contains a nonzero constantfunctional element. Assume $[x] \neq 0$ for some $x \in T$. Choose a variable $v \in \mathbf{V}$ so that $v$ does not occur in $x$. Then we have $\nvdash^{\mathrm{c}} c(v) \cdot x=0$ by $(\mathrm{C})$, so $c([v]) \cdot[x] \neq 0$ in $\mathfrak{T} / \cong$. By the Lemma above, $c([v]) \cdot[x]$ is a constant-functional element of $\mathfrak{T} / \cong$ which lies below $[x]$ in $\mathfrak{T} / \cong$. By Theorem $5, \mathfrak{T} / \cong$ is a representable relation algebra. It now follows, as in the previous proof, that if RRA $\vDash x=y$ then $\Xi_{\mathrm{SA}} \vdash^{\mathrm{c}} x=y$.

For the converse we need only show $(\mathrm{C})$ is sound. The proof is exactly the same as the proof that $(\mathrm{F})$ is sound, except that we must use the fact that every nonempty relation contains a nonempty constant function: any set containing a single ordered pair from the relation will do.

Finally, Venema's results cannot be improved in both ways simultaneously. This is due to the fact that there are semiassociative relation algebras in which every nonzero element contains a nonzero functional element, but these algebras are not even relation algebras, and hence are not representable. To get such an algebra, choose any finite Boolean algebra with four or more atoms. Choose one 
atom and call it 1 '. Define; on the atoms by setting, for all atoms $x, y$,

$$
x ; y= \begin{cases}x & \text { if } y=1, \\ y & \text { if } x=1, \\ 1^{\prime} & \text { if } x=y \neq 1, \\ \sum\left\{z: z \text { is an atom distinct from } 1^{\prime}, x, y\right\} & \text { if } x, y, 1^{\prime} \text { are distinct }\end{cases}
$$

and extend ; to all elements of the algebra as follows:

$$
u ; v=\sum\{x ; y: x, y \text { are atoms, } x \leq u, y \leq v\} .
$$

Let $\breve{u}=u$ for all $u$. These definitions yield a semiassociative relation algebra in which every atom is functional, but ; is not associative. This example is taken from $[9]$.

\section{References}

[1] L. H. Chin and A. Tarski, Distributive and modular laws in the arithmetic of relation algebras, Univ. California Publ. Math. (N.S.) 1 (1951), 341-384.

[2] S. Givant, Tarski's development of logic and mathematics based on the calculus of relations, in: Algebraic Logic, Colloq. Math. Soc. János Bolyai 54, North-Holland, Amsterdam 1991, 361-392.

[3] L. Henkin, J. D. Monk and A. Tarski, Cylindric Algebras, Part II, North-Holland, Amsterdam 1985.

[4] B. Jónsson, Varieties of relation algebras, Algebra Universalis 15 (1982), 273-298.

[5] - , The theory of binary relations, in: Algebraic Logic, Colloq. Math. Soc. János Bolyai 54, North-Holland, Amsterdam 1991, 245-292.

[6] B. Jónsson and A. Tarski, Boolean algebras with operators, Part II, Amer. J. Math. 74 (1952), 127-162.

[7] R. C. Lyndon, The representation of relational algebras, II, Ann. of Math. (2) 63 (1956), 294-307.

[8] R. D. Maddux, Some sufficient conditions for the representability of relation algebras, Algebra Universalis 8 (1978), 162-172.

[9] —, Topics in relation algebras, dissertation, Univ. of California, Berkeley 1978.

[10] - Some varieties containing relation algebras, Trans. Amer. Math. Soc. 272 (1982), 501526.

[11] - Necessary subalgebras of simple nonintegral semiassociative relation algebras, Algebra Universalis 27 (1990), 544-558.

[12] —, Pair-dense relation algebras, Trans. Amer. Math. Soc. 328 (1991), 83-131.

[13] - Introductory course on relation algebras, finite-dimensional cylindric algebras, and their interconnections, in: Algebraic Logic, Colloq. Math. Soc. János Bolyai 54, North-Holland, Amsterdam 1991, 361-392.

[14] - The origin of relation algebras in the development and axiomatization of the calculus of relations, Studia Logica 50 (1991), 421-455.

[15] R. D. Maddux and A. Tarski, A sufficient condition for the representability of relation algebras, Notices Amer. Math. Soc. 23 (1976), A-477.

[16] R. N. W. McKenzie, A general method for constructing elementary axioms for classes of representable structures, preprint, 1966. 
[17] J. D. Monk, On representable relation algebras, Michigan Math. J. 11 (1964), 207-210.

[18] —, Nonfinitizability of classes of representable cylindric algebras, J. Symbolic Logic 34 (1969), 331-343.

[19] A. Tarski and S. Givant, A Formalization of Set Theory without Variables, Amer. Math. Soc., 1987.

[20] Y. Venema, Two-dimensional modal logics for relational algebras and temporal logic of intervals, LP-89-03, Institute for Language, Logic, and Information, Univ. of Amsterdam, 1989.

[21] —, Many-dimensional modal logic, dissertation, Univ. of Amsterdam, 1992. 